\title{
Oral Manifestations of a Patient with Epidermolysis Bullosa
}

\author{
Nusrat Nazir* and Altaf Hussain Chalkoo \\ Department of Oral Medicine and Radiology, GDC Srinagar, India
}

Received: October 26, 2017; Published: November 01, 2017

*Corresponding author: Dr. Nusrat Nazir, Post graduate student ,Department of Oral Medicine and Radiology GDC Srinagar, Kashmir, Jammu \& Kashmir, India, Tel: 9697468033; Email: drnusratnaziromar@gmail.com

\begin{abstract}
Epidermolysis bullosa acquisita (EBA) is a chronic autoimmune bullous disease characterized by the presence of IgG and IgM antibodies at the level of basement membrane. It is rare in humans and animals with an incidence ranging from 0.2 to 0.5 new cases per million and per year. This dermatological condition is a severe autoimmune disease. Scarring of the extensor surfaces of the extremities, hands and feet are typical; milia occur frequently; and nails often become thick and dystrophic or are lost. The disorder affects both sexes equally and occurs in all racial and ethnic groups.
\end{abstract}

Keywords: Adherent fingers; Absent nails; Microstomia; Bullae; Preventive care

\section{Introduction}

Epidermolysis Bullosa (EB) is a group of rare inherited disorders, usually detected at birth or early childhood [1-4]. Köbner coined the term 'epidermolysis bullosa' in 1886, but even before this time, Legg and Brocq had already provided a clinical description of the disease. Epidermolysis bullosa characterized by extreme fragility of the skin and mucous membranes, which gives rise to the formation of blisters following minor trauma [5]. This dermatological condition is a severe autoimmune disease $[6,7]$. Scarring of the extensor surfaces of the extremities, hands and feet are typical; milia occur frequently; and nails often become thick and dystrophic or are lost. The disorder affects both sexes equally and occurs in all racial and ethnic groups [8]. Epidermolysis bullosa has been classified into three major types depending upon the histological level of tissue separation [9]:

I. Epidermolysis bullosa simplex is characterized by discontinuities in the epithelial keratinocyte layer;

II. Junctional epidermolysis bullosa involves separation within the basement membrane; and

III. Dystrophic epidermolysis bullosa is characterized by discontinuities in the underlying connective tissue.

Each type of EB has various subtypes and these may vary in severity [10]. Skin biopsies are needed for appropriate diagnosis and classification for affected subjects.

\section{Case Report}

A 12-year-old female patient diagnosed with severe generalized junctional EB was referred to the Department of oral medicine and
Radiology. The patient complaints of dental pain, halitosis, severe crustation of lips and limited mouth opening with ulcerations of buccal mucosa. The patient had one sister, aged 6 years old, who was unaffected by the disease. Her parents were also unaffected and were not consanguineous. Both sets of grandparents came from the nearby areas of same state. This type of illness had not previously appeared in the family Physical examination revealed generalized worn-out skin, blistering and scar formation, with blisters and vesicles present especially on the head and neck. The patient's few fingers were adherent, and her nails were absent (Figures 1 \& 2). Scar formation had resulted in the formation of microstomia (Figure 3). The patient's maximum mouth opening was $14 \mathrm{~mm}$. Clinical examination showed multiple missing teeth, decay and poor oral hygiene (Figure 4), due in part to a soft diet and hand contractures.

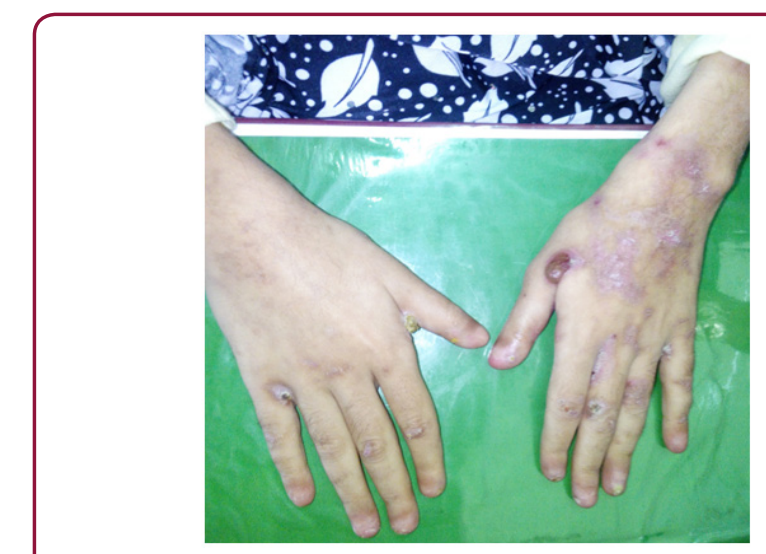

Figure 1: Nails absent. 


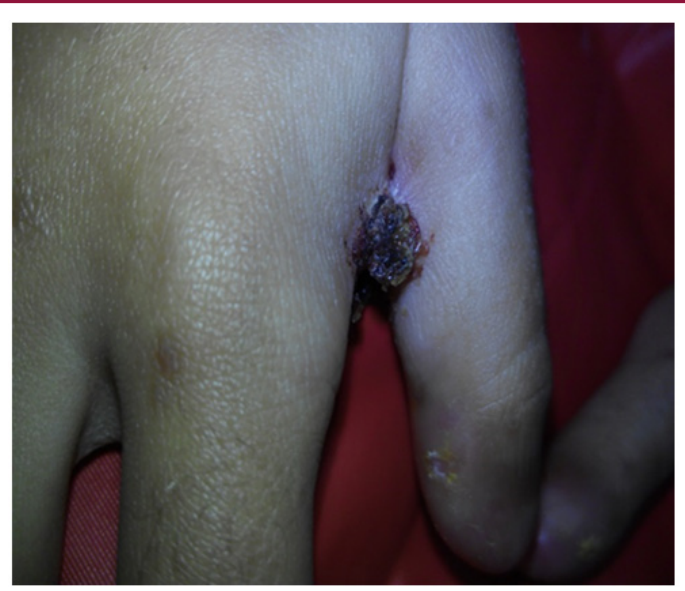

Figure 2: Fingers adherent.
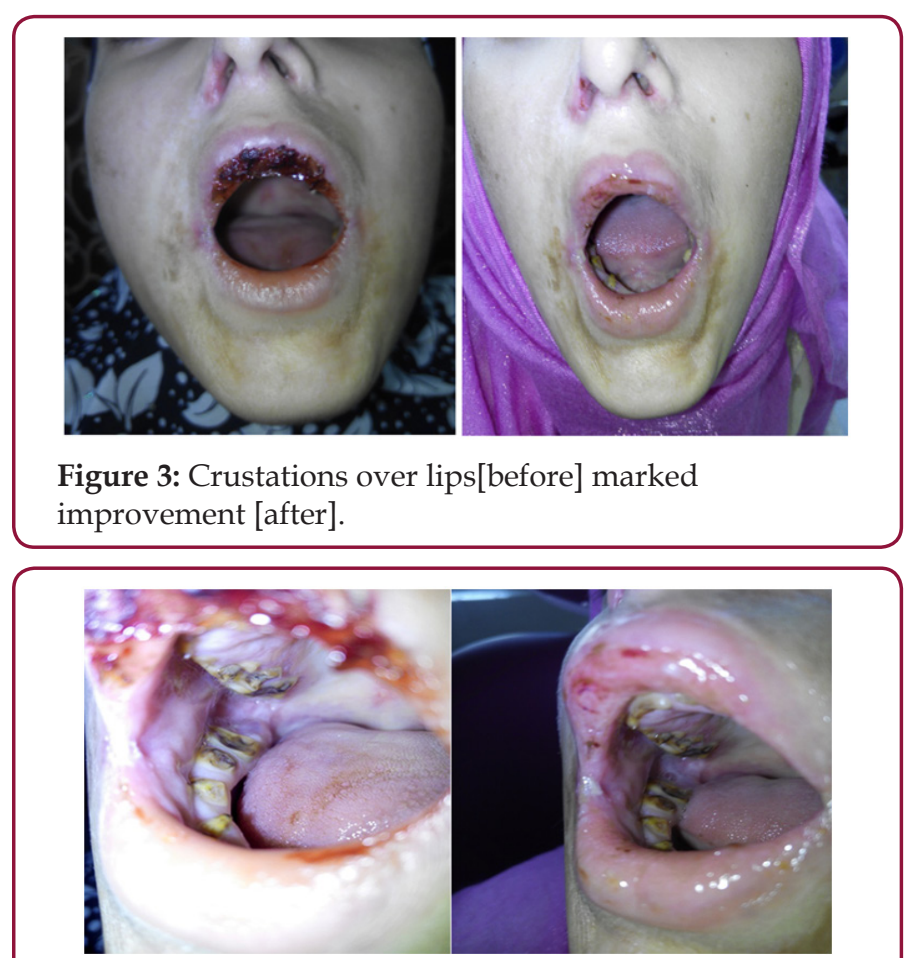

Figure 4: Severe ulcerations of buccal mucosa [before] marked improvement [after].

The patient was a case of junctional epidermolysis bullosa which was confirmed by histopathology. Skin biopsy from fresh bulla showed a large dermo-epidermal bulla with mild chronic inflammatory infiltrate in dermis. Direct immuno fluorescence showed IgG IgM deposition at the level of basement membrane; all the features were suggestive of junctional epidermolysis bullosa. The goal of treatment in the present case was to prevent blisters from forming and its resulting complications. Topical steroids and antibiotics were used to promote healing and prevent secondary infection of blisters. Since restoration of teeth was not possible due to severe crown destruction, extraction of few teeth was done in two sittings under local anaesthesia. The use of an aloe vera containing tooth gel at home was suggested to soothe the burning feeling affecting the gums. A mouthwash was also prescribed to help the salivary immune system protect the mucosal surfaces. In this case, minimal intervention has so far preserved the oral cavity and monthly topic fluoride application helped to control dental caries. The patient maintains continuous contact with the health team to avoid complex treatments. Follow-up is advised after every 3 months for the evaluation of oral status.

\section{Discussion}

EB is a group of rare genetically determined disorders characterized by the development of blisters following minor or insignificant trauma or traction to the skin or mucosal surfaces [11-13].Oral manifestations and dental involvement of EB vary in frequency, in severity and according to subtype. Dental management of individuals with EB has been reported previously by several authors $[14,15]$. Examinations have shown that the caries prevalence among individuals with dystrophic EB and junctional EB is significantly higher than among healthy people [16]. Similar observations have been made with respect to the prevalence of plaque and gingivitis. However, the patients examined in these studies show no significantly higher prevalence of Candida albicans, Lactobacillus casei or Streptococcus mutans [17]. Wright et al .reported that none of their patients with EB showed any evidence of a decreased salivary flow rate [18]. Despite the severe cutaneous and extracutaneous involvement associated with inherited EB, the above authors found no evidence to support the hypothesis of abnormal salivary function or mucosal immunity in this disease. Taken together, these findings suggest that the rampant dental caries seen in the various forms of EB are more likely to be attributable to non salivary factors, such as enamel involvement, soft-tissue alterations and/or diet. Dental caries have a complex pathogenesis and may be influenced by a variety of genetic and environmental factors. Childhood EBA is a very rare disease and none of the previously described patients had dental abnormalities as a clinical manifestation of this disease. Interestingly, defects in enamel maturation and/or mineralization have been reported in dystrophic and junctional epidermolysis bullosa. The Col17_/_ mice, a model to study non-Herlitzjunctional epidermolysis bullosa enamel hypoplasia, exhibit imperfect amelogenesis demonstrated by malformed enamel rods and irregular enamel matrix [19].

Similar changes are observed in some cases of epidermolysis bullosa caused by mutations in laminin 5, a6b4 integrin or collagen VII [20]. The tooth abnormalities due to type VII collagen deficiency investigated in Col7a1_/_ and COL7-rescued humanized mice may be attributed to poorly differentiated ameloblasts [21]. Defective enamel structure may provide a flourishing local environment for cariogenic bacteria by providing more adhesive and colonization potential and being more acid soluble. Hence, defective enamel appears to be a favoring factor for dental caries of childhood. Epidermolysis bullosa treatment is generally focused on support. Perforating the blisters contributes to accelerating the healing process and prevents continued lateral spread of the blisters. Currently, researchers are focusing their attention on gene and cell therapy, recombinant protein infusions, intradermal injections of allogenic fibroblasts and stem cell transplantation. Other developing therapies are directed toward the enhancement of wound healing and better quality of life for EB patients [22]. Some difficulties complicate the rehabilitation treatment of these 
patients, as the fragility of the mucosa and the microstomia. The lesions in the oral mucous membrane can be so severe that they interfere with nutrition as a result of the difficulty of food due to pain as in present case. Thus, the treatment of the EB patient must be modified in order to decrease the effects of local trauma to the tissues.

Oral ulceration due to trauma during dental manipulation is unavoidable, but it could be limited by the mucosa lubrication before any manipulation with hydrocortisone cream, triamcinalone or petroleum jelly [23]. In addition, the use of small-sized instruments, short-shaft dental burs and hand pieces with a small-sized head is indicated. Dentures and restorations should be carefully adapted and highly polished; secondary infections should be prevented with the use of oral antiseptics or using topical antibiotics on existing bullous lesions. During the dental treatment in outpatient settings, the administration of local anaesthesia to patients with EB may also cause blister formation and should therefore be avoided whenever possible. Nevertheless, when necessary, local anesthetics should be injected slowly and deeply into the tissues to prevent mechanical tissue separation and blistering. Owing to the difficulties related to the restorative dental treatment of these patients, the emphasis on the oral health management must be on prevention beginning at an early age. Regular application of topical and systemic fluoride, oral hygiene instructions and dietary guidelines minimize caries development and improves overall oral health.

\section{Conclusion}

Early dental management and preventive care to minimize caries development and to improve oral health is very important for patients with EB. Furthermore, they are often advised to reduce their consumption of cariogenic foods. Topical fluoride applications at regular intervals are also indicated. Ongoing patient monitoring must be stressed to minimize the need for restorative treatment in patients with EB.

\section{References}

1. Travis SP, McGrath JA, Tumbull AJ, Schofield OM, Chan O, et al. (1992) Oral and gastrointestinal manifestations of epidermolysis bullosa. Lancet 340: 1505-1506.

2. Wright JT, Fine JD, Johnson L (1994) Dental caries risk in hereditary epidermolysis bullosa. Pediatric Dentistry 16: 427-432.

3. Brooks JK, Bare LC, Davidson J, Taylor LS, Wright JT (2008) Junctional epidermolysis bullosa associated with hypo plastic enamel and pervasive failure of tooth eruption: Oral rehabilitation with use of an over denture. Oral Surgery Oral Medicine Oral Pathology Oral Radiology and Endodontics 105: e24-e28.

4. Lanschuetzer CM, Fine JD (2009) General aspects. In: Life with Epidermolysis Bullosa (EB). Etiology, Diagnosis, Multidisciplinary Care and Therapy. Fine JD, Hintner H (Eds.) Netherlands: Springer pp. 1-95.

5. Siañez-González C, Pezoa Jares R, Salas Alanis JC (2009) Congenital epidermolysis bullosa: a review. Actas Dermosifiliogr 100(10): 842-856.
6. Williams EF, Gannon K, Soon K (2011) The experiences of young people with epidermolysis bullosa simplex: a qualitative study. J Health Psychol 16(5): 701-710.

7. Komorowski L, Müller R, Vorobyev A, Probst C, Recke A, et al. (2013) Sensitive and specific assays for routine serological diagnosis of epidermolysis bullosa acquisita. J Am Acad Dermatol 68(3): e89-e95

8. Momeni A, Pieper K (2005) Junctional epidermolysis bullosa: a case report. International Journal of aediatric Dentistry15: 146-150.

9. Fine JD, Bauer EA, Briggaman RA, Carter DM, Eady RA, et al (1991) Revised clinical and laboratory criteria for subtypes of inherited epidermolysis bullosa. A consensus report by the Subcommittee on Diagnosis and Classification of the National Epidermolysis Bullosa Registry. Journal of the American Academy of Dermatology 24: 119-135.

10. Fine JD, McGrath J, Eady R (2000) Inherited epidermolysis bullosa comes into the new millennium: a revised classification system based on current knowledge of pathogenic mechanisms and the clinical, laboratory, and epidemiologic findings of large, well-defined patient cohorts. Journal of the American Academy of Dermatology 43: 135-137.

11. Carroll DL, Stephan MJ, Hays GL (1983) Epidermolysis bullosa-review and report of a case. J Am Dent Assoc 107: 749-751.

12. Fine JD, Johnson LB, Suchindran CM (1994) The National Epidermolysis Bullosa Registry. J Invest Dermatol 102: 545-565.

13. Gormley JW, Show CE (1976) Epidermolysis bullosa and associated problems in oral surgical treatment. J Oral Surg 34: 45-52.

14. Bohaty B, Spencer P, Dunlap C, Wandera A (1998) Epidermolysis bullosa: case report of appropriate classification of subtype because of an early dental exam. Journal of Clinical Pediatric Dentistry 22: 243-245.

15. Liu HH, Chen CJ, Miles DA (1998) Epidermolysis bullosa simplex: review and report of case. ASDC Journal of Dentistry for Children 65: 349-353.

16. Wright JT, Fine JD, Johnson L (1994) Dental caries risk in hereditary epidermolysis bullosa. Pediatric Dentistry 16: 427-432.

17. Harris JC, Bryan RA, Lucas VS, Roberts GJ (2001) Dental disease and caries related micro flora in children with dystrophic epidermolysis bullosa. Pediatric Dentistry 23: 438-443.

18. Wright JT, Childers NK, Evans KL, Johnson LB, Fine JD (1991) Salivary function of persons with hereditary epidermolysis bullosa. Oral Surgery, Oral Medicine and Oral Pathology 71: 553-559.

19. Vidal F, Baudoin C, Miquel C, Galliano MF, Uitto J, et al (1995) Cloning of the laminin alpha 3 chain gene (LAMA3) and identification of a homozygous deletion in a patient with Herlitz junctional epidermolysis bullosa. Genomics 30: 273-280.

20. Umemoto H, Akiyama M, Domon T, Nomura T, Shinkuma S, et al. (2012) Type VII collagen deficiency causes defective tooth enamel formation due to poor differentiation of ameloblasts. Am J Pathol 181: 1659-1671.

21. Hakki SS, Celenligil-Nazliel H, Karaduman A, Aysen K, Alp Usubutun, et al (2001) Epidermolysis bullosa acquisita: clinical manifestations, microscopic findings, and surgical periodontal therapy. A case report. J Periodontol 72: 550-558.

22. Kummer TR, Nagano HC, Tavares SS, Santos BZ, Miranda C (2013) Oral manifestations and challenges in dental treatment of epidermolysis bullosa dystrophica. J Dent Child 80(2): 97-100.

23. Stavropoulos F, Abramowicz S (2008) Management of the oral surgery patient diagnosed with epidermolysis bullosa: report of 3 cases and review of the literature. J Oral Maxillofac Surg 66: 554-559. 


Assets of Publishing with us
- Global archiving of articles

